\title{
Ferramenta para Tomada de Decisão Considerando a Interação dos Sistemas de Produção e o Meio Ambiente
}

\author{
Flávio A. Barrella \\ Cecilia M. Villas Boas de Almeida \\ Biagio F. Giannett। \\ Universidade Paulista - UNIP
}

\begin{abstract}
Resumo
Diagramas ternários são apresentados como ferramenta gráfica no auxílio da contabilidade ambiental e tomada de decisões ambientais baseadas na análise emergética. Além de inquestionáveis vantagens da interpretação gráfica sobre análise de tabelas, o uso dos diagramas ternários, largamente empregados para a avaliação da físico-química de diagrama de fases para sistemas de três componentes, permite o uso das propriedades do diagrama de fases para avaliação da dependência dos sistemas sobre entradas renováveis e não-renováveis e a avaliação do suporte ambiental para diluição e redução de emissões dos processos e da eficiência dos sistemas. A imediata visualização dos dados da contabilidade emergética permite a comparação de processos e sistemas com e sem serviços dos ecossistemas, para avaliar melhorias e acompanhamento do desempenho dos sistemas ao longo do tempo. Com a objetividade dos diagramas ternários, aspectos como a interação entre sistemas e a interação entre sistemas e meio ambiente podem ser prontamente reconhecidos e avaliados.
\end{abstract}

Palavras-chave

Sistemas de produção, ferramenta gráfica, diagrama ternário, sustentabilidade.

\section{Decision-making tool considering the interaction of the production systems and the environment}

\begin{abstract}
Ternary diagrams are presented as graphic tools to assist environmental accounting and environmental decisionmaking based on emergy analysis. Besides the unquestionable advantages of graphic interpretation over table analysis, the use of ternary diagrams, widely used for physical chemistry evaluation of three components systems, or three components phase diagrams, permits the use of phase diagrams properties to assess the dependence of the system upon renewable and non renewable inputs, the environmental support for dilution and abatement of process emissions and the system efficiency. The prompt visualization of the emergy accounting data makes possible to compare processes and systems with and without ecosystem services, to evaluate improvements and to follow the system performance over time. With the aim of ternary diagrams, aspects such as the interaction between systems and the interactions between systems and the environment can be readily recognized and evaluated.
\end{abstract}

Key words

Production systems, graphic tool, ternary diagram, sustainability. 


\section{INTRODUĈ̣̃O}

As empresas estão cada vez mais cônscias das conseqüências da deterioração ambiental e muitas delas têm incluído entre suas prioridades a proteção ao ambiente, aliada à qualidade e à segurança, como forma de assegurar a competitividade. A mudança de atitude das empresas em relação ao meio ambiente teve início nas áreas de produção e operação, onde há grandes oportunidades para reduzir o impacto de processos (SARKIS, 1995 e SHEN, 1995). Por um lado, o ambiente foi tomado como um desafio para a indústria (SHARMA e VREDENBURG, 1998; WALLEY e WHITEHEAD, 1994). Por outro lado, considerou-se que a relação ambiente-empresa, baseada em um gerenciamento ambiental efetivo, é capaz de trazer melhorias tanto para o ambiente como para a competitividade da empresa (SHEN, 1995; PORTER, 1995; HART, 1995). Os estudos realizados nos anos 90 pertencem ao segundo grupo e enfatizam os benefícios de um bom desempenho ambiental para operações e processos (GUPTA, 1995; SARKIS, 1995) e para as empresas como um todo (AZZONE e BERTELE, 1994; MAXWELL et al., 1997).

Apesar do grande número de estudos publicados na década de 90 , pouca atenção foi dada ao desenvolvimento de indicadores a serem utilizados nas tomadas de decisão que envolvem o aspecto ambiental, e a maioria dos indicadores está relacionada às áreas de produção, como indicadores para a escolha de equipamentos, métodos e práticas produtivas, rendimento de processos e desenvolvimento de produto (KLASSEN, 2000; SHRIVASTAVA, 1995; CONSTABLE et al., 2002).

A exploração de novas alternativas de operação para novos sistemas de produção ou para modificar os existentes exige investimento de capital e, por este motivo, a tomada de decisão nos meios produtivos foi inicialmente baseada em análises de viabilidade econômica. Heizer e Render (1996) propuseram o uso da análise financeira tradicional para a adoção de estratégias no desenvolvimento de produtos e processos. O mesmo método é também utilizado por vários autores, que sustentam ser necessário identificar os custos e benefícios provenientes dos investimentos voltados à redução do impacto ambiental (ALBERTI et al., 2000; SILVA, 2003).

Não obstante, o uso da análise financeira tradicional como critério para tomadas de decisão de caráter ambiental, apesar de ser geralmente aceito, não contempla benefícios e custos intangíveis. A avaliação do aspecto ambiental é bastante complexa e devem considerar um grande número de fatores, alguns dos quais não podem ser avaliados pela análise financeira tradicional, já que não há como estabelecer um valor monetário para custos e benefícios intangíveis como o valor de uma reserva natural ou o custo para o ambiente absorver poluentes. É, portanto, incoerente estabelecer a análise financeira como ferramenta para avaliar ganhos ou perdas ambientais, especialmente se a empresa tiver incluído a proteção ao ambiente entre suas estratégias de competitividade.

A avaliação ambiental com base na análise emergética leva em conta a taxa de exploração de reservas naturais, o uso eficiente destas reservas e a capacidade de suporte do meio ambiente (ODUM, 1996). Da definição de emergia como a memória da energia ou a energia total incorporada em um produto, processo ou serviço, os indicadores que resultam da análise emergética refletem tanto a contribuição do sistema econômico como a do meio ambiente (BROWN e ULGIATI, 2002). Estes indicadores são utilizados para avaliar as relações entre componentes do sistema produtivo e as reservas utilizadas para a obtenção do produto de interesse; dependem da fração de insumos renováveis e não renováveis e consideram os insumos disponíveis localmente e aqueles importados de fora do sistema. Obtémse, desta forma, informações valiosas sobre o desenvolvimento e a operação dos sistemas produtivos, levando em conta a capacidade de carga do ambiente. Pode-se ainda avaliar a eficiência termodinâmica do sistema, a qualidade dos fluxos de entrada e saída do sistema de produção e especialmente a interação entre o sistema e o ambiente em que este está inserido.

Desde a sua introdução, a análise emergética nos processos produtivos (ODUM, 1996) tem-se tornado cada vez mais importante no cenário mundial, sendo hoje estudada nas mais variadas instituições de pesquisa, quer sejam acadêmicas, quer sejam dos setores industriais ou governos. Encontram-se aplicações em vários setores, como na produção de energia elétrica (BROWN e ULGIATI, 2002; ULGIATI e BROWN, 2002; ODUM e PETERSON, 1996; ODUM, 2000; BJÖRKLUND et al., 2001), no tratamento de água (BROWN e McCLANAHAN, 1996; GEBER e BJÖRKUND, 2001; MARTIN, 2002), na fabricação de papel e celulose (LOPES et al., 2003), na produção agrícola (LEFROY e RYDBERG, 2003; PANZIERI et al., 2000; BASTIANONI e MARCHETTINI., 2000), no desenvolvimento urbano (SHU-LI e WAN-LIN, 2003; SHU-LI, 1998; HOWINGTON et al., 1997), nas relações com a economia tradicional (HORNBORG, 1998; CLEVELAND et al., 2000; TON et al., 1998; BROWN e ULGIATI, 1997), entre outros.

Estudos comparativos sobre meios de transporte de passageiros e carga e a utilização de combustíveis alternativos (RYDBERG e JANSEN, 2002; FEDERICI et al., 2003; B YRNE e POLONSKY, 2001) têm sido realizados 
com a utilização da análise emergética, e seus resultados têm demonstrado quais são as alternativas menos agressivas ao meio ambiente e suas conseqüências econômicas e de produção.

Vários estudos complementares têm, também, sido realizados (FATH et al., 2001; BAKSHI, 2000; BASTIANONI e MARCHETTINI, 1997; BASTIANONI e MARCHETTINI, 1996; PATTEN, 1995; JORGENSEN et al., 1995; POPP et al., 2001; MANOLIADIS, 2002; RONCHI et al., 2002; BARRERA e SALDÍVAR, 2002; SPANGENBERG, 2002; FEDERICI et al., 2003) consolidando a importância da análise e dos indicadores obtidos. Para diferentes setores e processos, novos índices e abordagens têm sido propostos (ULGIATI et al., 1995; POPP et al., 2001; MANOLIADIS, 2002; RONCHI et al., 2002; BARRERA e SALDÍVAR, 2002; SPANGENBERG, 2002; SEAGER e THEIS, 2002) expandindo o alcance da análise emergética, mas ao mesmo tempo gerando uma maior complexidade analítica.

Neste artigo, propõe-se a utilização de uma ferramenta gráfica, desenvolvida em $V i$ sual Basic for Applications (ROMAN, 2000; JACOBSON, 2002; ROOS, 1994), para representação dos índices resultantes da análise emergética de processos e de produtos. A ferramenta baseia-se nos diagramas de fases ou diagramas de três componentes, utilizados para representar propriedades de substâncias ou misturas de substâncias (CASTELLAN, 1984). A representação clara e uniforme dos indicadores em diagramas de fases permite visualizar os resultados da análise emergética e comparar sistemas diferentes ou diferentes momentos do mesmo sistema. A utilização dos diagramas permite ainda explorar suas propriedades já conhecidas (CASTELLAN, 1984) e acrescentar duas novas abordagens à análise emergética: introdução da sensibilidade emergética e da simergia. Demonstra-se que a interpretação dos resultados é facilitada com a padronização da ferramenta gráfica, auxiliando a tomada de decisão.

\section{REVISÃO TEÓRICA}

\section{Emergia e Indicadores}

Emergia é definida como a quantidade de energia necessária, de forma direta ou indireta, para obter um produto (bem ou serviço) em um determinado processo, sendo sua unidade expressa em seJ (joule de energia solar). O uso de uma única unidade para a qual são convertidos os diversos tipos de energia permite somar todas as contribuições de energia utilizadas para a obtenção de um determinado produto ou serviço. Define-se a transformidade como a quantidade de energia solar empregada, diretamente e/ou indiretamente, na obtenção de um joule de um determinado produto (bem ou serviço), sendo sua unidade expressa em seJ/J (joule de energia solar por Joule). A transformidade fornece uma medida da concentração de emergia e pode ser considerada como um indicador de qualidade. Os valores de emergia e transformidade dependem do material e da energia utilizados nas diversas etapas necessárias para a obtenção do produto ou serviço e, por este motivo, variam de acordo com a matéria-prima selecionada, com o tipo de energia empregado na produção e com a eficiência do sistema produtivo.

\section{$\Lambda$ tomada de decisão nos meios produtivos viabilidade econômica.}

As fontes de recursos que compõem qualquer processo podem ser divididas em três classes: renováveis, nãorenováveis e provenientes da economia. Esta divisão é fundamental para o estudo emergético e a inter-relação do setor ou processo com o meio ambiente. Na Figura 1 observa-se que fluxos de entrada em um sistema de produção são divididos em reservas não-renováveis $(\mathrm{N})$, serviços e produtos do ambiente renováveis $(\mathrm{R})$ e fluxos provenientes da economia $(\mathrm{F})$. Os recursos renováveis (R) são retirados do ambiente e, como o próprio nome diz, têm a capacidade de renovação temporal e espacial mais rápida que o seu consumo. Encontram-se dentro dessa classe a energia solar, dos ventos, da chuva, etc. Os recursos não-renováveis $(\mathrm{N})$ são armazenados na natureza, porém seu consumo é mais rápido do que a sua capacidade de renovação. Dentro dessa classe encontram-se fontes de recursos como carvão, petróleo, florestas, água potável, etc. Os recursos pagos (F) - vindos da economia - são associados a bens e serviços ou a recursos provenientes de outras regiões fora dos limites do sistema estudado. A Figura 1 mostra um diagrama simplificado em que são identificados os fluxos de entrada $\mathrm{R}, \mathrm{N}$ e $\mathrm{F}$ e o fluxo de saída do produto $\mathrm{Y}$.

Os fluxos de recursos identificados na figura 1 permitem calcular diferentes índices que, por sua vez, podem ser de grande utilidade para analisar ou monitorar um 
sistema. Neste artigo serão definidos apenas os indicadores empregados: o rendimento emergético (EYR), o investimento emergético (EIR), o impacto ambiental ou índice de carga ambiental, o índice de sustentabilidade (SI ou EIS) e o percentual de emergia renovável (\%R).

Calcula-se o rendimento emergético pela relação entre a emergia total do produto $(\mathrm{Y}=\mathrm{R}+\mathrm{N}+\mathrm{F})$ e a emergia de entrada do setor econômico, que não é fornecida gratuitamente pelo meio ambiente (Equação 1). Este índice reflete a habilidade do processo de utilizar recursos locais (ULGIATI e BROWN, 2002), mas não diferencia recursos renováveis de não-renováveis.

$$
E Y R=\frac{Y}{F}=\frac{R+N+F}{F}
$$

O investimento emergético é a relação entre a emergia fornecida pelo sistema econômico e a fornecida diretamente pelo meio ambiente ao sistema estudado, quer seja renovável ou não-renovável (Equação 2).

$$
E I R=\frac{F}{N+R}
$$

Define-se a carga ambiental como a relação entre a soma da emergia de entrada proveniente do sistema econômico e do recurso local não-renovável e a emergia do recurso local renovável (Equação 3). Este índice fornece um balizamento dos serviços ambientais num sistema e mostra um balanço entre os investimentos locais renováveis e não-renováveis. Um valor alto de ELR pode indicar um estresse de utilização dos recursos renováveis locais.

$$
E L R=\frac{N+F}{R}
$$

O índice de sustentabilidade é obtido da relação entre o rendimento emergético e o índice de carga ambiental (Equação 4). O conceito de sustentabilidade está atrelado à maximização de EYR (rendimento) e a minimização de ELR (impacto), ou seja, o máximo do aproveitamento do investimento com um mínimo de estresse dos recursos locais.

$$
S I=\frac{E Y R}{E L R}=\frac{\frac{Y}{F}}{\frac{N+F}{R}}
$$

Finalmente, o percentual de energia renovável é a parcela de emergia total processada de um determinado sistema que provém de fontes de recursos renováveis (Equação 5).

$$
\% R=\frac{R \times 100}{R+N+F}=\frac{R \times 100}{Y}
$$

Figura 1: Diagrama de fluxos emergéticos, onde aparecem os fluxos de entrada de recursos renováveis $(R)$, não-renováveis $(N)$ e provenientes da economia $(F)$ e o fluxo de saída do produto $(Y)$.

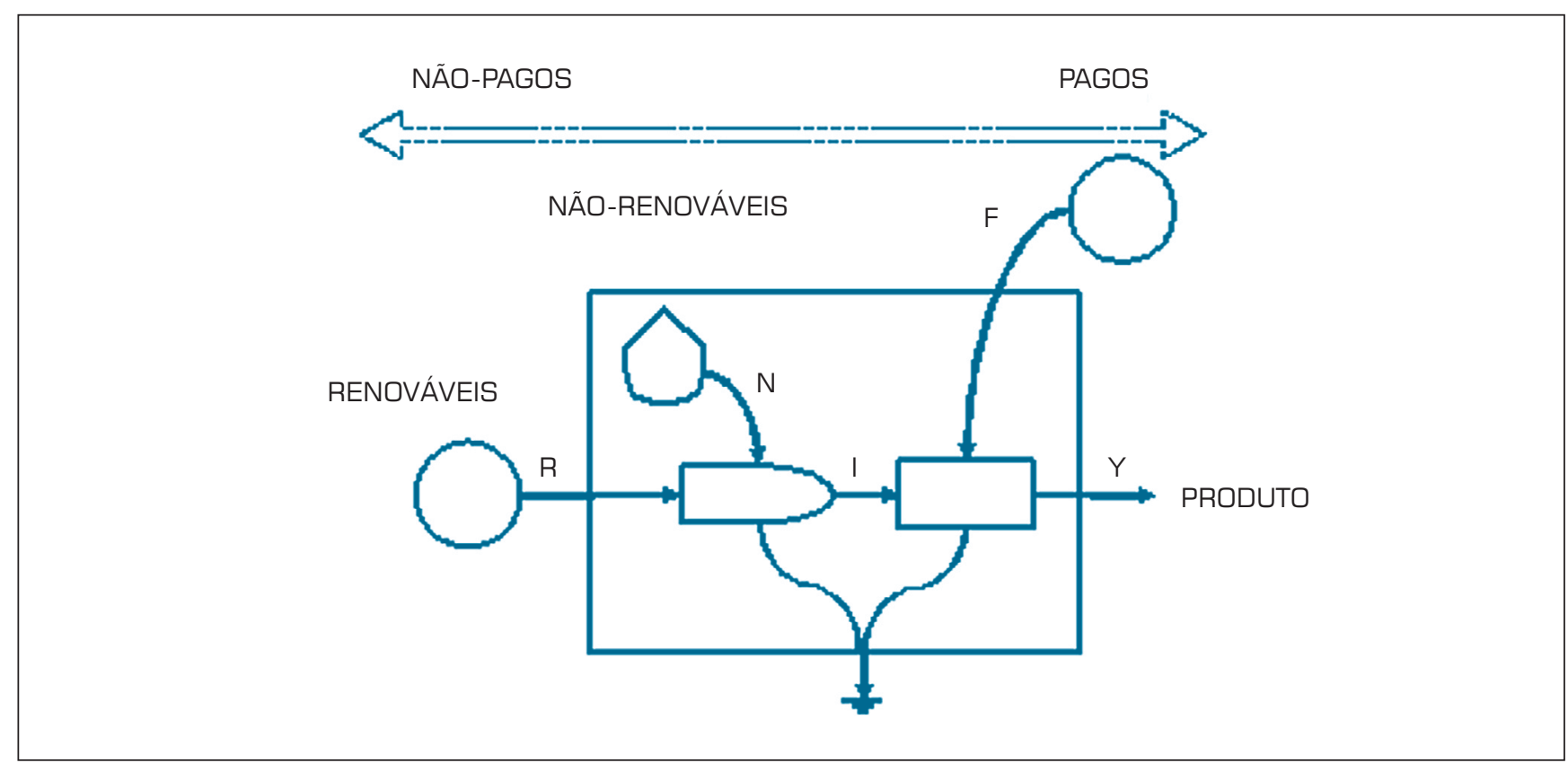




\section{Diagrama de Fases}

O método de análise para o estudo de fases em misturas de substâncias proposto por Gibbs e Roozeboon fornece um método gráfico muito eficiente quando temos três variáveis para a descrição de um processo ou de um sistema (CASTELLAN, 1984).

O diagrama triangular é definido como um sistema de coordenadas a partir de um triângulo eqüilátero cuja altura é igual à unidade. Cada uma das alturas é associada a uma das variáveis, conforme apresentado na Figura 2a. Assim, um ponto qualquer inscrito no triângulo pode ser definido a partir de um conjunto de três valores de coordenadas. Estes são relacionados com um sistema de coordenadas cartesianas com os valores positivos de abscissas coincidentes com a mediana do lado da base do triângulo e a ordenada sobreposta à altura e a origem na interseção da altura e a base do triângulo.

Uma importante propriedade dos diagramas é que a soma das coordenadas triangulares das variáveis é igual à altura do triângulo. Tomando-se a altura do triângulo igual à unidade, teremos que a soma das coordenadas é igual a um, conforme mostrado na Figura 2b. Uma segunda propriedade se refere à combinação de sistemas. Quando combinamos dois sistemas cujas composições são representadas pelos pontos $\mathrm{P}$ e $\mathrm{Q}$, na Figura $2 \mathrm{c}$, a composição da mistura resultante será representada por um ponto $\mathrm{X}$ sobre o segmento PQ. Finalmente, outra propriedade importante é que todos os sistemas representados pelos pontos de uma linha que passa por um dos vértices do triângulo possuem dois de seus componentes na mesma razão, conforme apresentada na Figura 2d.

\section{METODOLOGIA}

Para este trabalho foi desenvolvido um aplicativo em dois arquivos Excel, com rotinas escritas em Visual Basic for Applications, onde foram correlacionadas a cada um dos eixos do diagrama triangular uma das fontes de recursos (renováveis, não-renováveis e pagas). Um dos arquivos da ferramenta é utilizado para preparação dos dados de entrada, tendo capacidade para a inclusão de até 10 processos com até 50 itens por processo (Figura 3a). Outro arquivo da ferramenta captura esses dados, faz os cálculos necessários e os aplica em um diagrama triangular, apresentando um ponto para cada processo, produto ou sistema. Ainda estão disponíveis linhas auxiliares que podem ser mostradas no diagrama para facilitar a análise.

Figura 2: Diagrama Triangular, representação de: (a) sistema de coordenadas triangulares; (b) propriedade da soma das coordenadas; (c) propriedade da combinação de sistemas; (d) propriedade da proporcionalidade.
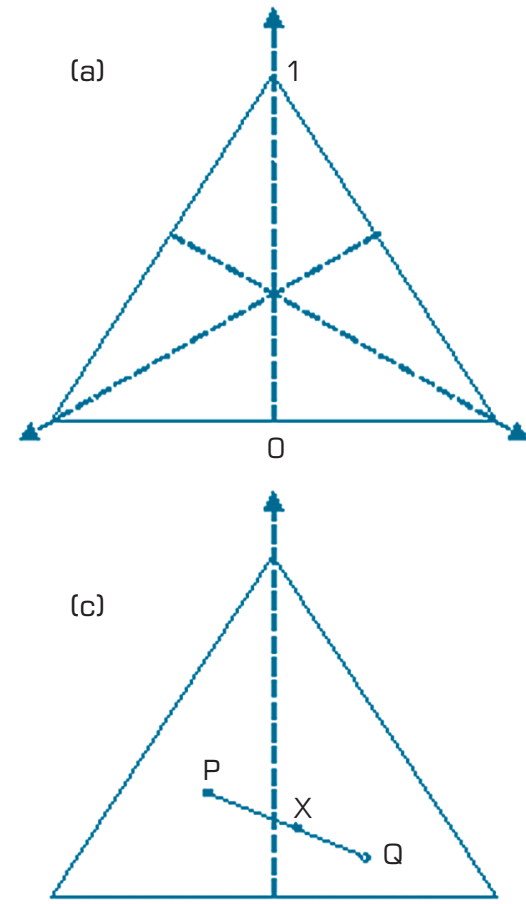
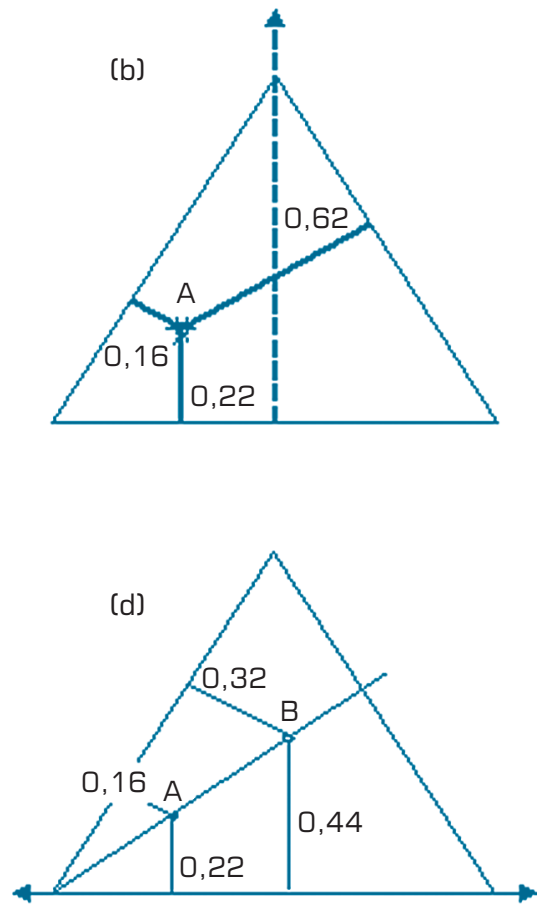
A ferramenta permite a apresentação de até 10 processos ou produtos simultaneamente (Figura $3 b$ ). Cada ponto pode ser habilitado ou inibido para que não se apresente no diagrama. Ainda pode ser selecionada a apresentação dos pontos com tamanho fixo ou com tamanho proporcional à diferença da ordem de grandeza dos valores de emergia total do produto de cada um, isto é, quanto maior o valor de emergia do produto maior o tamanho do ponto apresentado. Isto fornece uma idéia das ordens de grandezas dos vários produtos ou processos representados.

Para explorar as propriedades dos diagramas triangulares, esta correlação foi feita com valores relativos de cada fonte de recurso e não com os seus valores absolutos, o que não altera os valores dos índices estudados. da componente F. Dessa forma podemos associar à linha de F os valores dos índices EYR e EIR. Segundo Brown e Ulgiati (2002), valores de EYR abaixo de 5 devem ser considerados como indicativos de fontes de energia secundária e materiais primários como cimento e aço. Fontes de energia primária usualmente têm valores de EYR maiores que 5. Além disso, processos que tenham rendimento emergético (EYR) menor que 2 não fornecem nenhuma contribuição que possa ser considerada como fonte de energia e atuam como produtos de consumo ou etapas na transformação das fontes de energia reais. Análise semelhante é feita com a componente $\mathrm{R}$ em relação ao índice ELR. Ainda segundo Brown e Ulgiati (2002), valores baixos de ELR (cerca de 2) são indicativos de relativo baixo impacto ambiental (ou processos que usam uma grande área de ambiente local para diluir o impacto). Valores de ELR maiores que 10 são indicativos de relativa concentração de impacto ambiental, e aqueles entre 3 e 10 podem ser considerados moderados. Valores extremamente altos de ELR podem ser o resultado de investimentos em um ambiente relati-

Escolheu-se para as coordenadas triangulares, como sendo o eixo das fontes de recursos renováveis $(\mathrm{R})$ coincidente com as ordenadas do sistema cartesiano, o eixo das fontes de recursos não-renováveis $(\mathrm{N})$ partindo da mediana do lado direito do triângulo e passando pelo vértice à esquerda da base do triângulo. $\mathrm{O}$ eixo de fontes de recursos pagos (F) parte da mediana do lado esquerdo do triângulo e passa pelo vértice à direita da base do triângulo. Dessa forma, verifica-se que qualquer processo ou sistema pode ser representado por um ponto no diagrama, dado que qualquer processo ou sistema conta com estas três fontes de recursos.

O programa permite, ainda, o traçado de linhas para estabelecer limites ou identificar aspectos com maior facilidade:

Linhas de Fontes de Recursos: A ferramenta permite a apresentação de 3 linhas ( $\mathrm{R}, \mathrm{N}$ e F) para indicação dos valores constantes de cada uma das fontes de recursos. Essas linhas podem ser apresentadas independentemente e os valores escolhidos pelo usuário, numa faixa entre 0 e 1. As linhas de fontes de recursos são segmentos de retas e paralelas aos três lados do triângulo. Com a sua utilização pode-se avaliar e comparar dois processos em relação ao uso de recursos (Figura 4a). Com o uso da ferramenta gráfica, em que se considera o valor de $\mathrm{Y}$ unitário (valor normalizado do produto final do processo), os índices EYR e EIR podem ser calculados a partir vamente pequeno com entradas derivadas de energias não-renováveis muito concentradas.

Na Figura 4a, pode-se observar que os sistemas A e B utilizam a mesma porcentagem de recursos pagos, apesar de utilizarem diferentes quantidades de recursos renováveis e não-renováveis.

Linhas de Sustentabilidade: A ferramenta permite a apresentação de 3 linhas (SIi, SIj e SIk), conforme apresentadas na Figura 4b, para indicação dos valores constantes de sustentabilidade. Essas linhas podem ser apresentadas independentemente e os valores escolhidos pelo usuário numa faixa entre 0 e infinito. As linhas de sustentabilidade partem do vértice $\mathrm{N}$ e cruzam o lado oposto ao vértice, permitindo assim dividir o diagrama em áreas de sustentabilidade para comparar a sustentabilidade de processos.

Segundo Brown e Ulgiati (2002) valores de SI menores que 1 são indicativos de produtos ou processos que não são sustentáveis a longo prazo. Sistemas com valores maiores que 1 indicam produtos e processos que dão contribuições sustentáveis para a economia. Sustentabilidade em médio prazo pode ser caracterizada por um SI entre 1 e 5 enquanto produtos e processos com sustentabilidade a longo prazo têm SI maiores.

Linhas de Sensibilidade Emergética: Existe a possibilidade de apresentação de três linhas $\left(\mathrm{S}_{\mathrm{R}}, \mathrm{S}_{\mathrm{N}}\right.$ e $\left.\mathrm{S}_{\mathrm{F}}\right)$ conforme Figura 5, sobre as quais pode-se acompanhar a 
Figura 3: Telas dos Aplicativos. (a) Entrada de dados. (b) Diagrama ternário e os menus de seleção.

(a)

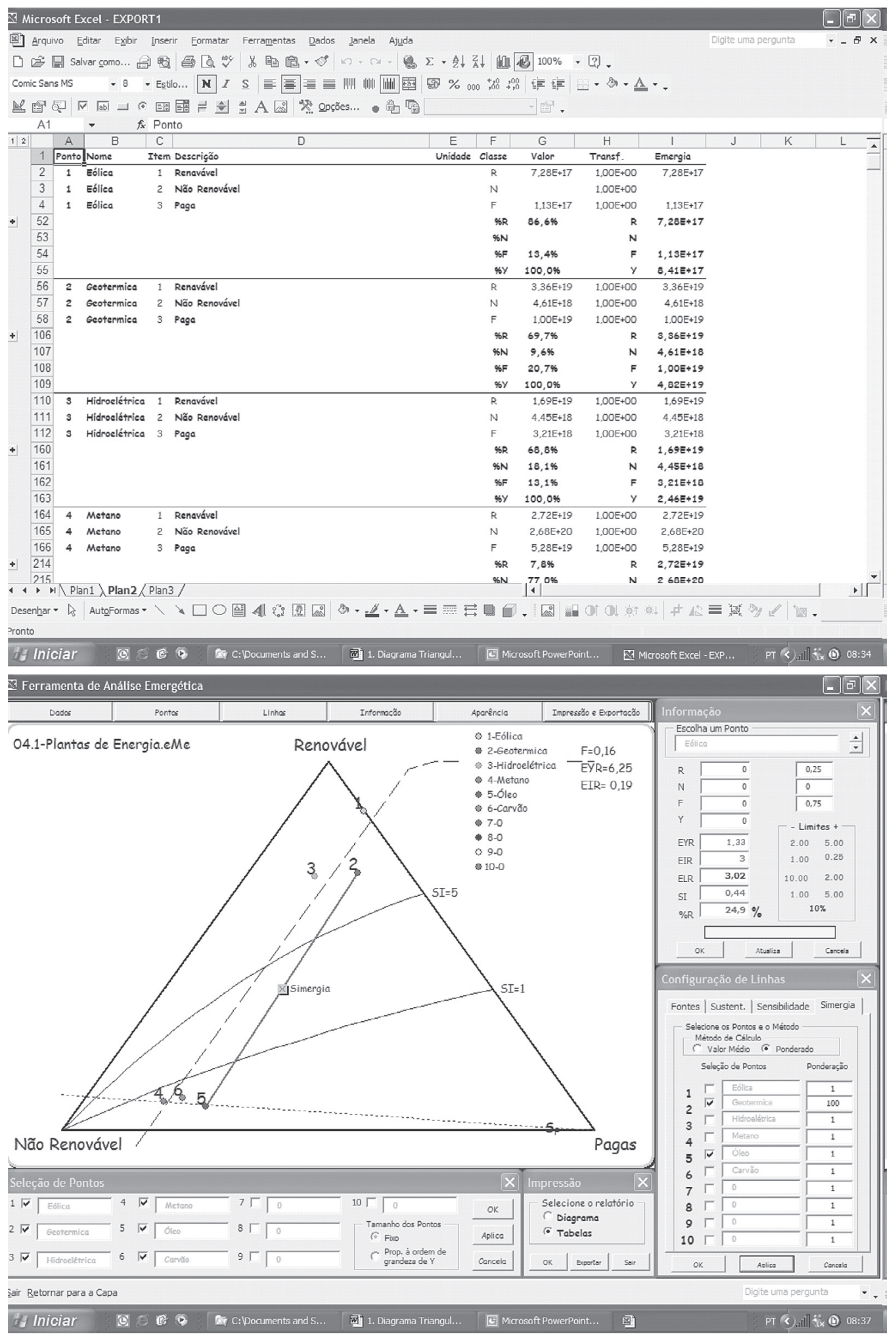


variação da fonte de recurssos considerada ( $\mathrm{R}, \mathrm{N}$ e F). Ao longo de cada linha as outras duas fontes de recursos mantêm a mesma proporção (propriedade da proporcionalidade). O usuário pode escolher o ponto desejado para que as linhas de sensibilidade emergética o contenham. O seu principal uso é para a análise da evolução de processos.
Ponto Simérgico: Ainda há a possibilidade de apresentação de um ponto, que é denominado de ponto simérgico ou simplesmente simergia, que é definido pela composição de dois ou mais pontos que estejam apresentados no diagrama (Figura 6). O ponto simérgico pode ser calculado pela associação direta (valor médio) das emergias de cada classe de fonte de recursos para cada ponto

Figura 4: Diagrama triangular com (a) as três linhas de recursos e (b) as três linhas de sustentabilidade $\mathrm{SI}_{\mathrm{i}}=1, \mathrm{SI}_{\mathrm{i}}=2$ e $\mathrm{SI}_{\mathrm{k}}=5$.

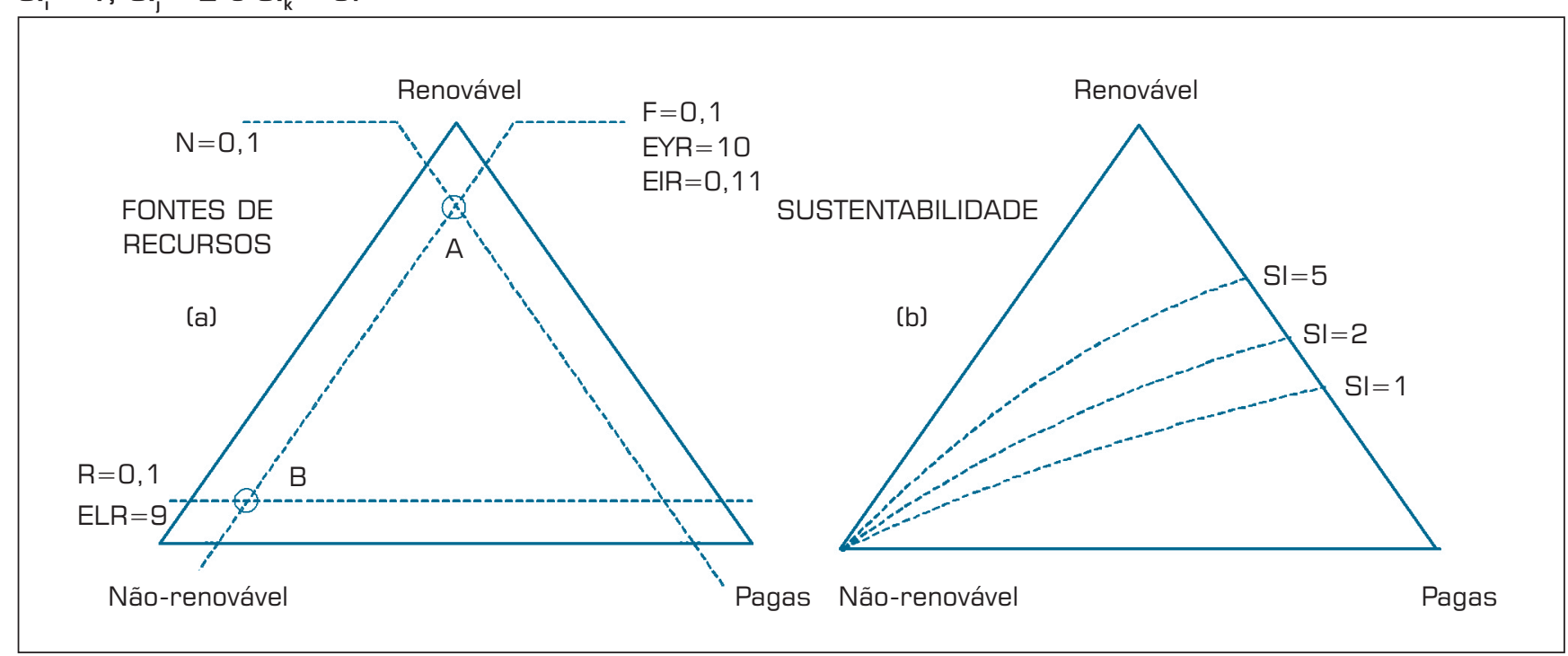

Figura 5: Diagrama triangular onde aparecem as três linhas de sensibilidade emergética do ponto A1 em relação às fontes de recursos renovável $\left[S_{R}\right]$, não renovável $\left[S_{N}\right]$ e pagas $\left[S_{F}\right]$ e as três linhas de sensibilidade - emergética para o ponto $A 2$ após um aumento na fonte de recursos pagos.

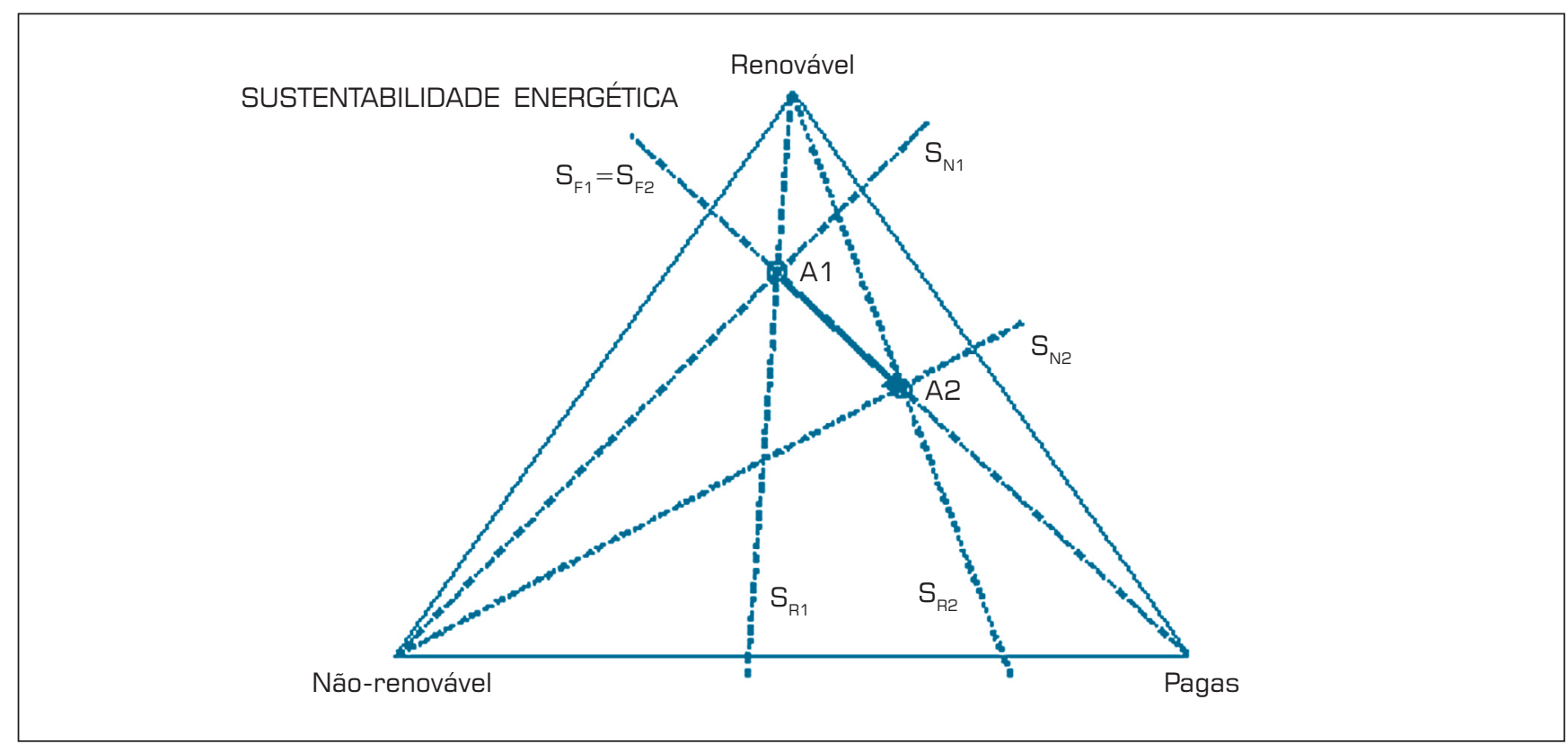


considerado, ou ainda, por um fator de ponderação aplicado aos termos do valor médio, escolhido pelo usuário e selecionado de uma tabela de ponderação. O ponto simérgico está associado à propriedade de composição de misturas do diagrama de fases e é utilizado para determinar as características da combinação de dois ou mais processos. A sua aplicação fica condicionada ao entendimento de que os fluxos de recursos dos vários componentes de um ponto simérgico são independentes. Se os fluxos não forem independentes deverá ser considerado somente o maior deles.

Esta última possibilidade de análise é útil quando se estuda o efeito de vários processos sobre o ambiente, por exemplo, o impacto de um parque industrial ou de um setor como o metalúrgico ou o de fornecimento de energia elétrica, como veremos a seguir. O cálculo e a visualização imediata do impacto total de um setor só é possível com a utilização da ferramenta e da propriedade que possibilita a determinação do ponto simérgico.

\section{APLICAC̣̃̃O}

Para efeito ilustrativo será abordado o caso de geração de energia elétrica em seis plantas com seis diferentes fontes de recursos (eólica, geotérmica, hidroelétrica, metano, óleo e carvão). Os valores de emergia dos fluxos de recursos consumidos pelas plantas e a produção anual de energia elétrica de cada planta são mostrados na Tabela 1 e foram retirados da literatura (BROWN e ULGIATI, 2002; ULGIATI e BROWN, 2002). Os artigos foram escolhidos de forma a dar um cunho didático e elucidar as possibilidades de tratamento dos dados permitidas pela ferramenta.

Figura 6: Diagrama triangular onde aparecem o ponto simérgico $\mathrm{X}$ e as linhas que o ligam aos pontos que 0 compõem A e B.

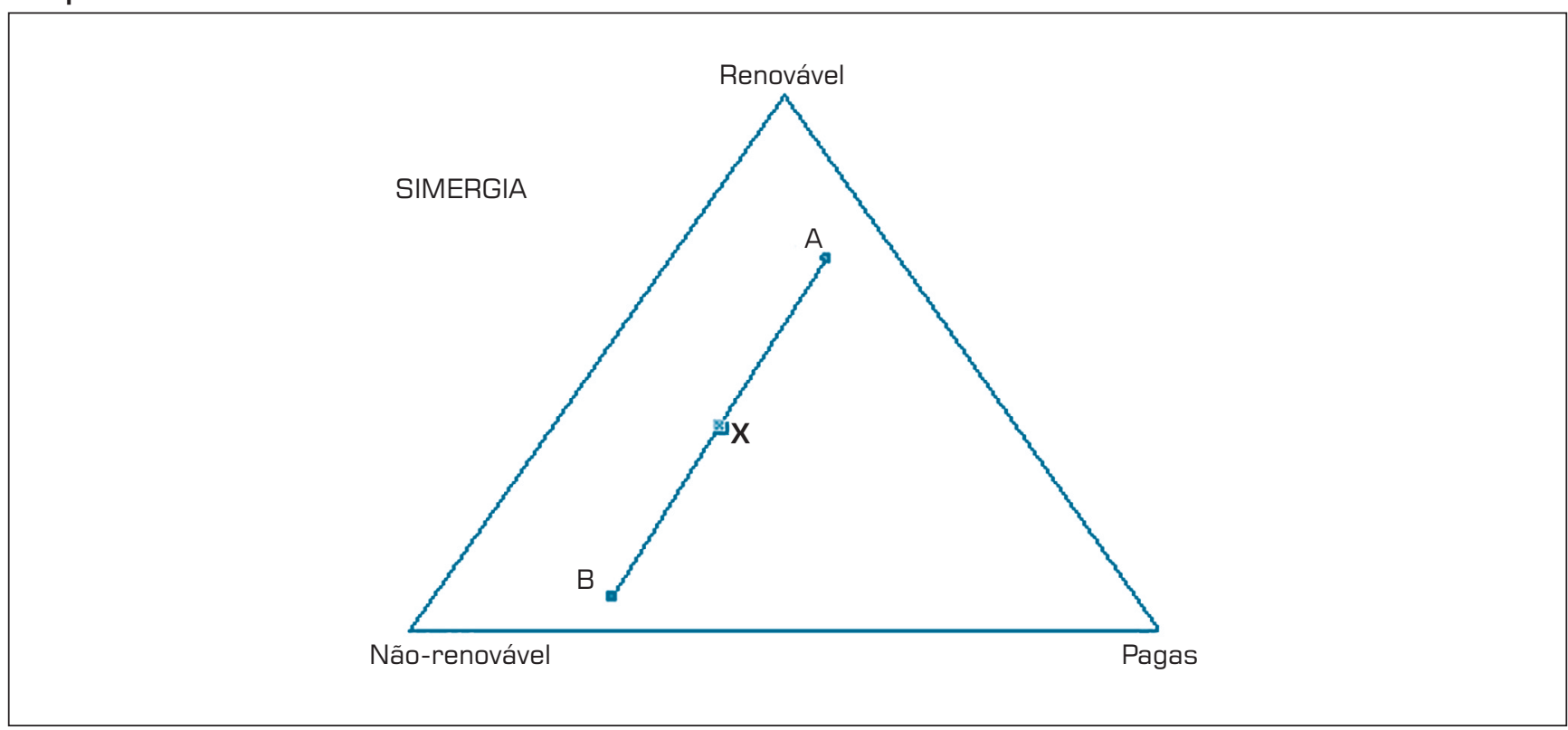

Tabela 1: Valores de emergia para plantas de energia elétrica (BROWN e ULGIATI, 2002).

\begin{tabular}{|l|c|c|c|c|}
\hline $\begin{array}{c}\text { PLANTA } \\
\text { DE ENERGIA }\end{array}$ & $\begin{array}{c}\text { ENERGIA PRODUZIDA } \\
\text { (W/ano) }\end{array}$ & $\begin{array}{c}\text { RENOVÁVEL } \\
\text { (seJ) }\end{array}$ & $\begin{array}{c}\text { NÃO-RENOVÁVEL } \\
\text { (sed ) }\end{array}$ & $\begin{array}{c}\text { PAGA } \\
\text { (sed ) }\end{array}$ \\
\hline Eólica & $2,5 \times 10^{6}$ & $7,28 \times 10^{17}$ & $0,00 \times 10^{00}$ & $1,13 \times 10^{17}$ \\
\hline Geotérmica & $20 \times 10^{6}$ & $3,36 \times 10^{19}$ & $4,61 \times 10^{18}$ & $1,00 \times 10^{19}$ \\
\hline Hidroelétrica & $85 \times 10^{6}$ & $1,69 \times 10^{19}$ & $4,45 \times 10^{18}$ & $3,21 \times 10^{18}$ \\
\hline Metano & $171 \times 10^{6}$ & $2,72 \times 10^{19}$ & $2,68 \times 10^{20}$ & $5,28 \times 10^{19}$ \\
\hline Óleo & $1280 \times 10^{6}$ & $3,12 \times 10^{20}$ & $3,32 \times 10^{21}$ & $1,13 \times 10^{21}$ \\
\hline Carvão & $1280 \times 10^{6}$ & $3,68 \times 10^{20}$ & $3,05 \times 10^{21}$ & $7,63 \times 10^{20}$ \\
\hline
\end{tabular}


A partir da Tabela 1 foram calculados os índices EYR, EIR, ELR e SI para todas as plantas de energia, conforme apresentados na Tabela 2.

Observa-se na Tabela 1 que a produção de energia elétrica nas plantas de óleo e carvão é igual (1280MW) e o uso dos recursos renováveis e não-renováveis equivalente. Há uma pequena diferença nos recursos vindos da economia. Porém, a análise da tabela não permite uma escolha entre as duas plantas de energia, nem no que concerne ao investimento econômico e nem quanto ao uso de recursos naturais. de fontes de recursos. Observa-se que os pontos 4,5 e 6 (metano, carvão e óleo) encontram-se sobre a linha de fonte de recursos $\mathrm{R}=0,08$, que indica a utilização de $8 \%$ de recursos renováveis. Considerando-se que o índice ELR (Tabela 2), que pode ser descrito pela quantidade de recursos renováveis utilizados, é a medida do impacto ambiental, pode-se considerar que as três plantas teêm características de agressão semelhantes ao meio ambiente, o que pode ser prontamente observado no diagrama.

Observa-se também na Figura 7c que todas as plantas têm sua componente de recursos pagos em torno de $16 \%$ como indica a linha $\mathrm{F}=0,16$. É interessante notar que, tanto nas plantas que causam um grande impacto ambiental quanto naquelas cujo impacto é menor, o investimento econômico é muito semelhante. Considerando-se que o índice EYR (Tabela 2) está fortemente atrelado à quantidade de recursos pagos, tem-se uma medida do rendimento ambiental do investimento. Todas as plantas têm esse índice entre 4,21

Os dados da Tabela 1 foram inseridos no aplicativo de entrada de dados. No diagrama da Figura 7a apresentamos os seis pontos obtidos, que representam as seis plantas de energia. Verifica-se que os pontos estão distribuídos no diagrama, localizando assim cada planta em uma área de sustentabilidade. Muitas vezes é útil, no estudo de um setor ou grupo de processos ou produtos, isolar um subconjunto para que a análise fique mais fácil e objetiva de ser realizada. Na Figura $7 b$ apresenta-se outra possibilidade da ferramenta: Pode-se selecionar, por exemplo, o subgrupo das plantas eólica, geotérmica e hidroelétrica, com a opção de apresentação dos pontos com tamanho proporcional à relação da ordem de grandeza de Y. Caso a decisão recaia sobre implantação de plantas de energia que não utilizam combustíveis fósseis é possível compará-las isoladamente. É fácil visualizar que as plantas geotérmicas e hidroelétricas têm um valor de Y maior que a eólica.

O diagrama da Figura 7c mostra a utilização das linhas e 7,47, indicando estarem na faixa de fontes de recursos primárias ou quase primárias.

No diagrama da Figura 7d estão apresentadas todas as plantas de energia e também as linhas de sustentabilidade com valores $\mathrm{SI}=1$ e $\mathrm{SI}=5$. Verifica-se que as três plantas de produção que não utilizam combustível à base de carbono estão em uma região acima da linha de SI $=5$ e as três plantas de combustíveis à base de carbono em uma região abaixo da linha de $\mathrm{SI}=1$. Aqui a opção torna-se clara quando se deseja produzir energia elétrica de forma sustentável a longo prazo.

No diagrama da Figura 7e estão apresentadas as plantas metano e óleo e também a linha de sensibilidade emergética em relação à fonte de recurso paga $(F)$. Verifica-se que as plantas estão sobre a mesma linha, significando que as proporções das fontes de recursos $\mathrm{N}$ e $\mathrm{R}$ para os dois pontos não variam, mas somente a proporção da componente da fonte de recurso de F. Isto nos indica que entre uma ou outra opção a diferença está no investimento econômico.

Tabela 2: Índices para as seis plantas de energia elétrica, calculados com auxílio da ferramenta gráfica.

\begin{tabular}{|l|l|l|l|l|}
\hline PLANTA DE ENERGIA & EYR & EIR & ELR & SI \\
\hline Eólica & 7,47 & 0,16 & 0,16 & 47,9 \\
\hline Geotérmica & 4,82 & 0,26 & 0,43 & 11,0 \\
\hline Hidroelétrica & 7,65 & 0,15 & 0,45 & 16,8 \\
\hline Metano & 6,59 & 0,18 & 11,79 & 0,56 \\
\hline Óleo & 4,21 & 0,31 & 14,26 & 0,30 \\
\hline Carvão & 5,48 & 0,22 & 10,36 & 0,53 \\
\hline
\end{tabular}


No diagrama da Figura $7 \mathrm{f}$ estão apresentadas as plantas eólica e hidroelétrica com as linhas de sustentabilidade que as contém ( $\mathrm{SI}_{\text {eólica }} \cong 48$ e $\mathrm{SI}_{\text {hidroelétrica }} \cong 17$, Tabela 2 ). Além disso, é apresentado também o ponto simérgico considerando um fator de ponderação de 8:1 que é a relação de produção de energia elétrica em watts entre elas (20MW para a hidroelétrica e 2,5MW para a eólica). A linha de sustentabilidade que contém o ponto simérgico tem o valor $\mathrm{SI}_{\mathrm{E} \text { Hi }}=21$. A partir do cálculo de SI das plantas eólica e hidroelétrica em conjunto, podese tomar decisões sobre o tipo de planta a investir, verificar qual o efeito da implantação de uma nova planta ou do aumento da capacidade de produção de uma delas sobre a sustentabilidade do sistema em longo prazo.

Considerando que as plantas estudadas por Ulgiati e Brown sejam representativas do setor de geração de energia da Itália, pode-se ponderar o cálculo do ponto simérgico pela matriz de produção de energia elétrica italiana para o ano de 2002 (PUBBLICAZIONE， 2003), conforme mostrado nas Figuras $7 \mathrm{~g}$ e $7 \mathrm{~h}$. Observa-se na Figura $7 \mathrm{~g}$ que a produção de energia elétrica italiana depende fortemente das usinas termoelétricas, o que se reflete na posição do ponto simérgico na Figura $7 \mathrm{~h}$.

\section{DISCUSSÃo}

A análise emergética é uma ferramenta poderosa com a qual se podem analisar produtos, processos e setores industriais e sua interação com o meio ambiente. Entretanto, a comparação baseada somente em valores e tabelas pode não deixar inteiramente clara a amplitude das diferenças entre os elementos comparados. Por exemplo, embora as plantas de energia metano, óleo e carvão (Tabela 2), tenham índices de impacto ambiental semelhantes (ELR, Tabela 2), a observação da Figura 7c mostra ainda que as três plantas utilizam a mesma porcentagem de recursos renováveis e que um aumento de investimento econômico não traria vantagens com relação à sustentabilidade do sistema. Da mesma forma ao se avaliar as plantas eólica e hidroelétrica com relação ao rendimento emergético $\left(\mathrm{EYR}_{\text {eólica }}=7,47 \mathrm{e}\right.$ $\left.\mathrm{EYR}_{\text {hidroelétrica }}=7,65\right)$ e ao investimento emergético $\left(\mathrm{EIR}_{\text {eólica }}=0,16\right.$ e EIR $\left.\mathrm{E}_{\text {hidroelérrica }}=0,15\right)$, verifica-se que ambas têm valores semelhantes para os dois indicadores (Tabela 2). A observação destes dois sistemas na Figura $7 \mathrm{c}$ indica que estão sobre a reta de $\mathrm{F}=0,13$, o que mostra que o investimento econômico, nos dois casos, é praticamente o mesmo. Pela posição no gráfico, podese ainda afirmar que a planta eólica tem características de sustentabilidade superiores à hidroelétrica.

Um outro aspecto interessante que pode ser ressaltado é a característica da relação entre as fontes de recursos. Por exemplo, a Tabela 1 mostra os valores de R, N e F para as plantas de metano e óleo. Com o auxílio da linha de sensibilidade emergética, mostrada na Figura 7e, fica evidente que a relação entre as fontes de recursos renovável e não-renovável mantêm-se de uma planta para outra, enquanto só há variação na relação da componente de fonte de recurso paga. Isto indica que, para uma mesma utilização dos recursos locais renováveis ou não, necessitamos de mais recursos pagos na planta de óleo do que na de metano.

\section{A ferramenta combina o rigor científico - da análise emergética com a praticidade da utilização dos diagramas ternários, já comprovada em várias áreas do conhecimento.}

É comum encontrar situações em que processos, como os exemplificados, representam a evolução de um processo ao longo do tempo, indicando a tendência do processo e fornecendo base para ações quer sejam de reforço, quer sejam corretivas do mesmo.

A utilização do ponto simérgico é uma abordagem que só é possível com a utilização da ferramenta gráfica e que se torna bastante interessante ao permitir a obtenção das características de todo um conjunto. Pode-se aplicar esse conceito para, por exemplo, a análise setorial de uma determinada área da economia ou, ainda, um pólo industrial geograficamente confinado. Para ilustrar essa análise, considerando as plantas de energia na Figura $7 \mathrm{~h}$, como representativas do parque energético italiano, cada uma com o seu processo produtivo próprio, verificamos que os valores do índice de sustentabilidade de cada uma delas têm valores muito diferentes (Tabela 2). A análise do índice de sustentabilidade para o conjunto das seis plantas, calculada a partir da média $\left(\mathrm{SI}_{\text {Médi }} \mathrm{O}=12,9\right)$ ou da média ponderada considerando a produção de cada planta $\left(\mathrm{SI}_{\text {média ponderada }}=\right.$ 3,66 ), não tem significado a não ser o matemático. Por outro lado, com a utilização do cálculo do ponto simérgico, que considera a produção de cada uma das unidades na utilização dos recursos $\mathrm{R}, \mathrm{N}$ e $\mathrm{F}$, pode-se calcular o valor do índice de sustentabilidade do sistema $\left(\mathrm{SI}_{\text {sistema }}=0,59\right)$. A utilização deste conceito pode, 
Figura 7: Diagrama Triangular: (a) plantas de energia elétrica; (b) subgrupo das plantas que não utilizam combustiveis fósseis; (c) linhas de R constante e F constante; (d) linhas de sustentabilidade; (e) linha de sensibilidade emergética em relação ao fluxo F; (f) ponto simérgico; (g) matriz energética da ltália; (h) ponto simérgico das plantas de energia elétrica ponderadas pela matriz de produção italiana.

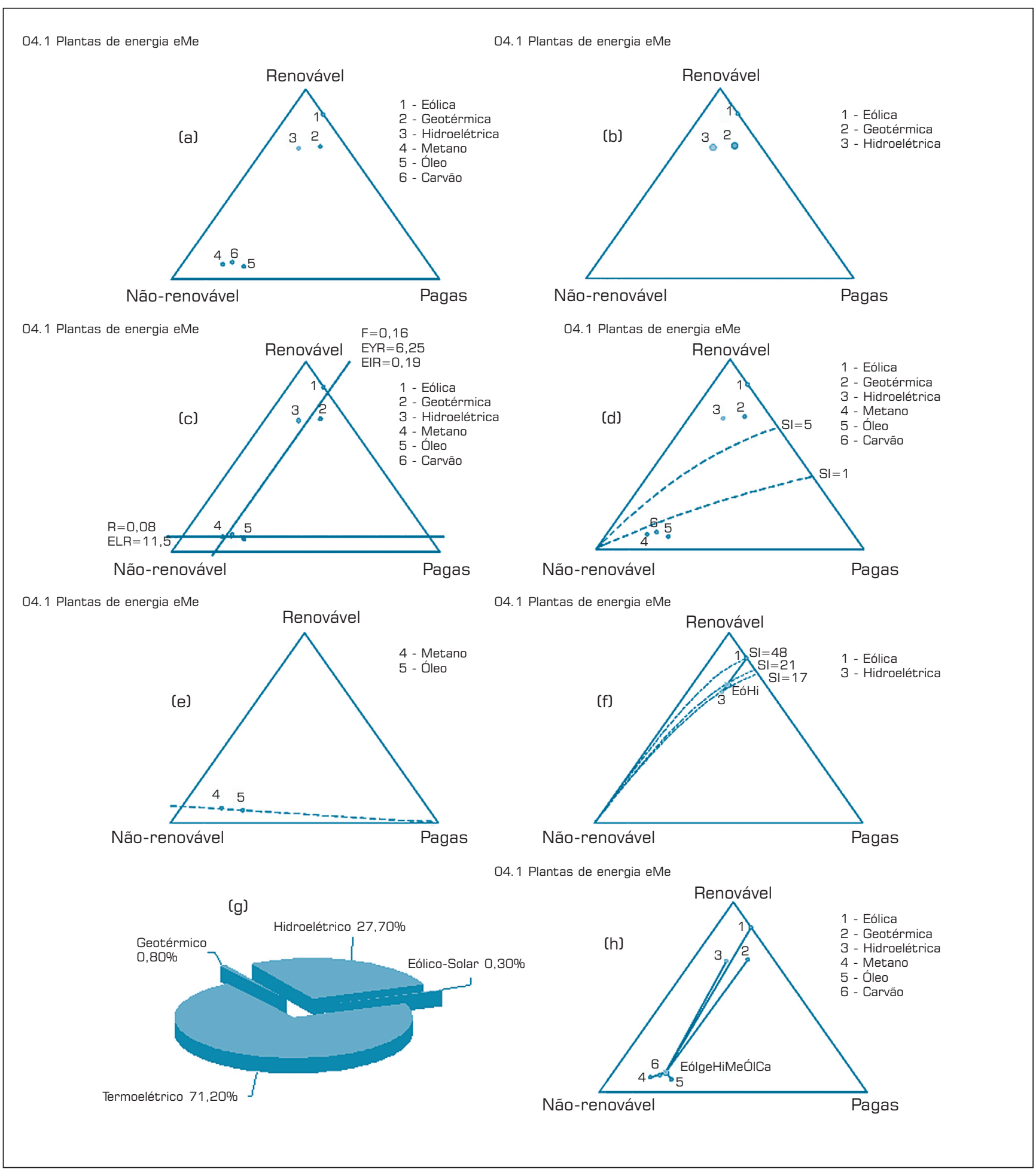

Fonte: Relatório anual do governo italiano (2003). 
por exemplo, auxiliar o legislador a definir regras, normas e leis para regulamentações de setores.

\section{CONCLUSÃO}

A metodologia e a ferramenta gráfica apresentadas vêm auxiliar e complementar os modelos hoje empregados para a tomada de decisões. Pode-se verificar que as possibilidades de análise e simulação com base na contabilidade emergética utilizando o suporte do diagrama triangular são infindáveis. Por meio da análise de sustentabilidade pode-se simular ações, como o aumento de investimento ou a troca de matéria-prima, e prever os possíveis impactos ambientais gerados por estas ações. Evita-se, assim, que a tomada de decisões no âmbito da produção gere efeitos não desejados, tanto para o ambi- ente como para a própria empresa.

A ferramenta combina o rigor científico da análise emergética com a praticidade da utilização dos diagramas ternários, já comprovada em várias áreas do conhecimento, como a físico-química, a mineralogia e a metalurgia. A representação gráfica facilita a interpretação dos dados para engenheiros e administradores, auxiliando e acelerando as tomadas de decisão, e é uma ferramenta útil para mostrar avanços e melhorias de produtos e processos para investidores, podendo ser, também, utilizada para facilitar a interpretação de relatórios públicos ambientais.

Administradores, do setor público e privado, e empresários poderão aplicar a ferramenta para gerenciar e monitorar recursos disponíveis de forma compatível com a vocação ecológica e com a capacidade real para sua sustentabilidade.

\section{Artigo recebido em 16/12/2003 Aprovado para publicação em 20/11/2004}

- Referências Bibliográficas

ALBERTI, M.; CAINI, L.; CALABRESE, A.; ROSSI, D. Evaluation of the costs and benefits of an environmental management system. International Journap. 4455-4466, 2000

AZZONE, G.; BERTELE, U Exploiting green strategies for competitive adavantages. Long Range Planning, v. 27, n. 6 , p. 69 81,1997

BAKSHI B.R. A thermodynamic framework for ecologically concious process system engineering. Computers and Chemical Engineering. 24 , p. 1767-1773, 2000

BARRERA-ROLDÁN A.; SALDIVARVALDÉS A. Proposal and application of a Sustainable Development Index. Ecological Indicators, 2, p. 251-256 2002.

BASTIANONI S.; MARCHETTINI N. Emergy/exergy ratio as measure of the level of organization of systems. Ecological Modelling, 99 p. 33-40, 1997.
BASTIANONI S.; MARCHETTINI N. Environmental cost and state: the problem of adiabaticity in the emergy value. Ecological Modelling, 90 , p. $33-37,1996$

BASTIANONI S.; MARCHETTINI N. The problem of co-production in environmental accounting by emergy analysis. Ecological Modelling, 129, p. 187-193, 2000.

BJÖRKLUND J.; GEBER U.; RYDBERG T. Emergy analysis of municipal wastewater treatment and generation of electricity by digestion of sewage sludge. Resources, Conservation and Recycling, 31, p. 293-316, 2001.

BROWN M. T.; ULGIATI S. Emergy Evaluations and Environmental Loading of Electricity Production Systems. J Cleaner Prod, 10, p. 321$334,2002$.

ROWN M.T. MCCLANAHAN T.R. Emergy analysis perspectives of Thailand and Mekong River dam proposals. Ecological Modelling, 91, p. 105-130, 1996.
BROWN M.T.; S. ULGIATI. Emergybased indices and ratios to evaluate sustainability: monitoring economies and technology toward environmentally sound innovation. Ecological Engineering, 9, p. 51-69, 1997.

BYRNE M. R.; POLONSKY M. J., Impediments to consumer adoption of sustainable transportation, International journal of Operations \& Production Management, 21 (2001) 1521-1538.

CASTELLAN G.W., "Físico Química 1", Livros Técnicos e Científicos Editora S.A., (1984) 363-365

CLEVELAND C.J.; KAUFMANN R.K.; STERN D.I., Aggregation and the role of energy in the economy, Ecological Economics, 32 (2000) 301-317.

CONSTABLE, J. C. DAVID; CURZONS, ALAN D.; CUNNINGHAM, VIRGINIA L. Metrics to 'green' chemistry which are the best?, Green Chemistry, 4 (2002) 521-527.
FATH B.D.; PATTEN B.C.; CHOI J.S., Complementarity of Ecological Goal Functions. Journal Theor. Biol., 208, p. $493-506,2001$

FEDERICI M.; ULGIATI S.; VERDESCA D.; BASOSI R. Efficienc and sustainability indicators for passenger and commodities transportation systems. Ecological Indicators, 3, p. 155-169, 2003.

GEBER U.; BJÖRKLUND J. The relationship between ecosystem services and purchased input in Swedish wastewater treatment systems - a case study. Ecological Engineering, 18, p. 39-59, 2001.

HORNBORG A. Comentary: Towards en ecological theory of unequal exchange: articulating world system theory and ecological economics. Ecological Economics, 25, p. 127-136, 1998.

GUPTA, M. C. Environmenta management and its impact on the operations functions. International Journal of Operations \& Production Management, v. 6, n. 4, p.6-16, 1995. 


\section{- Referências Bibliográficas}

HART, S. A natural resource based view of the firm. Academy of Management Review, v. 20, n. 4, p. 986-1014, 1995.

HEIZER, J.; RENDER B. Production and operation management: strategic and tactical decisions. Prentice Hall, Englewood Cliffs, NJ, 1996.

HOWINGTON T.M.; BROWN M.T.; WIGGINGTON M. Effect of hydrologic subsidy on selforganization of a constructed wetland in Central Florida. Ecological Engineering, 9, p. 137-156, 1997.

JACOBSON, REED; Microsoft Excel 2002 Visual Basic for Applications Passo a Passo, Makron Books, 326, 2002

JORGENSEN S.E.; NIELSEN S.N.; MEJER H. Emergy, environ, exergy and ecological modelling. Ecological Modelling, 77, p. 99-109, 1995.

KLASSEN, R. D. Exploring the linkage between investment in manufacturing and environmental technologies. International Journal of Operations \& Production Management, v. 20, n. 2, p. 127-147, 2000.

LEFROY E.; RYDBERG T. Emergy evaluation of three cropping systems in southwestern Australia. Ecological Modelling, 161, p. 195 211,2003

LOPES E. et al. Application of Life Cycle Assessment to the Portuguese Pulp and Paper Industry. J. Cleaner Prod., 11, p. 51-59, 2003.

MANOLIADIS, ODYSSEUS G. Development of ecological indicators-a methodological framework using compromise programming. Ecological Indicators, 2, p. $169-176,2002$
MARTIN J.F. Emergy valuation of diversions of river water to marshes in the Mississipi River Delta. Ecological Engineering, 18, p. 265-286, 2002.

MAXWELL, J., ROTHENBERG, S., BRISCOE, F.; MARCUS, A. Green schemes: corporate environmental strategies and their implementation", California Management Review, v. 39, n. 3, p. 118-34, 1997.

ODUM H.T. Environmental Accounting - EMERGY and Environmental Decision Making. Ed. John Wiley \& Songs Ltd., 370, 1996.

ODUM H.T. Emergy evaluation of an OTEC electrical power system, Energy, 25, p. 389-393, 2000.

ODUM H.T.; PETERSON N. Simulation and evaluation with energy systems blocks. Ecological Modelling, 93, p. 155-173, 1996

PANZIERI M.; MARCHETTINI N.; HALLAM T.G. Importance of the Bradhyrizobium japonicum symbiosis for the sustainability of a soybean cultivation. Ecological Modelling, 135, p. $301-310,2000$.

PATTEN B.C., Network integration of ecological extremal principles: exergy, emergy, power, ascendency, and indirect effects. Ecological Modelling, 79, p.75-84, 1995.

POPP J.; HOAG D.; HYATT D. E. Sustainability indices with multiple objectives. Ecological Indicators, 1 , p. $37-47,2001$.

PORTER M.; LINDE VAN DER C. Green and Competitive, Harward Business Review, p. 120-134, Set/Out, 1995.

PORTER M.; LINDE VAN DER C. Toward a new conception of the environment competitiveness relantionship, J. Econ. Perspect., 9, p. 97-118, 1995 .
PUBBLICAZIONE della Presidenza de Consiglio dei Ministri - Dipartimento per l'Informazione e l'Editoria Direttore: Mauro Mais Coordinamento editoriale Augusta Busico - Stampa e diffusione Istituto Poligrafico e Zecca dello Stato S.p.A - Salário Roma, 2003

ROMAN, STEVEN. "Desenvolvendo Macros no Excel". Ciência Moderna, 569,2000

RONCHI E.; FEDERICO A. e MUSMEC F. A system oriented integrated indicator for sustainable development in Italy. Ecological Indicators, 2, p. 197-210, 2002.

ROOS, NELSON. "Visual Basic for Windows Versão 3.0", Makron Books, 390, 1994

RYDBERG T.; JANSÉN J. Comparison of horse and tractor traction using emergy analysis, Ecological Engineering, 19, p. 13-28, 2002.

SARKIS, J. "Manufacturing strategy and environmental conciousness", Technovation, v. 15 , n. 2, p. 79-97, 1995.

SHARMA, S. e VREDENBURG, $\mathrm{H}$. "Proactive corporate environmental strategy and the development of competitively valuable organizational capabilities". Strategic Management Journal, v. 19, n. 8, p. 729-753, 1998.

SEAGER T. P.; THEIS T. L. A uniform definition and quantitative basis for industrial ecology. Journal of Cleaner Production, 10, p.225-235, 2002.

SHEN, T. T. Industrial Pollution Prevention, Berlin: Springer-Verlag, 1995.

SHRIVASTAVA, P. Environmental technologies and competitive advantages. Strategic Management Journal, v. 19, n. 3, p. 183-200, 1995.

SHU-LI HUANG. Urban ecosystems, energetic hierarchies, and ecological economics of Taipei metropolis Journal of Environmental Management, 52, p. 39-51, 1998.
SHU-LI HUANG; WAN-LIN HSU. Materials flow analysis and emergy evaluation of Taipei's urban construction. Landscape and Urban Planning, 63, p. 61-74, 2003.

SILVA B.A. Contabilidade e Meio Ambiente: Considerações teóricas e práticas sobre o controle dos gastos ambientais. São Paulo: Annablume Editora, 2003.

SPANGENBERG J.H.. Environmental space and the prism of sustainability: frameworks for indicators measuring sustainable development, Ecological Indicators, 2, p. 295-309, 2002.

TON S.; ODUM H.T.; DELFINO J.J. Ecological-economic evaluation of wetland management alternatives, Ecological Engineering, 11, p. 291-302, 1998.

ULGIATI S.; BROWN M. T. Quantifying the environmental support for dilution and abatement of process emissions - The case of electricity production. J Cleaner Prod, 10, p. 335$348,2002$.

ULGIATI S.; BROWN M.T., Monitoring patterns of sustainability in natura and man-made ecosystems. Ecological Modelling, 108, p. 23-36, 1998.

ULGIATI S; BROWN M.T; S BASTIANONI; N. MARCHETTINI, Emergy-based indices and ratios to evaluate the sustainable use of resources. Ecological Engineering, 5, p. 519-531, 1995.

ULGIATI S.; ODUM H.T.; BASTIANONI S. Emergy use, environmental loading and sustainability: $\mathrm{Na}$ emergy analysis of Italy, 73, p. 215 268,1994

WALLEY, N. e WHITEHEAD, B. "It's not easy being green". Harvard Business Review, vol 72 , n. 3, p. 4652, 1994. 


\section{- Agradecimentos}

Os autores agradecem à Vice-Reitoria de Pesquisa e Pós Graduação pelo apoio a este trabalho e ao Dr. Ian Olsson (Oskarhamn, Suécia) pelas proveitosas discussões e valiosas sugestões. A ferramenta apresentada ainda está em fase inicial de desenvolvimento e, portanto, deverá ser testada o maior número de vezes possíveis, permitindo que as suas funcionalidades possam ser revisadas e alteradas, otimizando as tomadas de decisões que nela se apóiam. Os autores disponibilizam a ferramenta para os interessados e agradecem qualquer colaboração que possa surgir, com sugestões e críticas, que possa de alguma forma ajudar a aprimorá-la.

- Sobre os autores

\section{Flávio A. Barrella}

Universidade Paulista - UNIP

Mestrando do curso de Engenharia de Produção - Produção e Gestão Ambiental - Produção e Meio Ambiente e Ciência e Produção mais Limpa.

End.: Rua Lenine Severino, 89 - São Paulo - SP - CEP 05353-230

Tel.: (11) 3714-6992

Cel.: (11) 9948-9885

E-mail: flavio.barrella@uol.com.br

\section{Cecília M. Villas Boas de Almeida}

Universidade Paulista - UNIP

Profa. Dra. do curso de mestrado de Engenharia de Produção - Produção e Gestão Ambiental - Produção e Meio Ambiente e Ciência e Produção mais Limpa.

End.: Rua Dr. Bacelar, 1212 - Saúde - São Paulo - SP - CEP 04028-002

Tel.: (11) 5586-4127

E-mail: cmvbag@unip.br

\section{Biagio F. Giannetti}

Universidade Paulista - UNIP

Prof. Dr. do curso de mestrado de Engenharia de Produção - Produção e Gestão Ambiental - Produção e Meio Ambiente e Ciência e Produção mais Limpa.

End.: Rua Dr. Bacelar, 1212 - Saúde - São Paulo - SP - CEP 04028-002

Tel.: (11) 5586-4127

E-mail: biafgian@unip.br 